\title{
An Image Correction Algorithm of Spinning Mirror Display
}

\author{
Yupeng Chen ${ }^{1, \text { a }}$, Yulin Xiang ${ }^{1, \mathrm{~b}}$, Xiang Liü ${ }^{1, \mathrm{c}}$, Hao Zhan ${ }^{1, \mathrm{~d}}$, Li Zhang ${ }^{1, \mathrm{e}}$ \\ 1. Huazhong University of science and technology, Hubei, Wuhan, 430000, China \\ acypeng2008@gmail.com, boringfor@outlook.com, cliuxiang@hust.edu.cn, dU201311207@hust \\ .edu.cn, ${ }^{\mathrm{z}} \mathrm{zlmse@mail.hust.edu.cn}$
}

Keywords: 3D image algorithm, Volumetirc display image source, Spinning mirror display, image correction.

Abstract. This paper presents an new image correction algorithm for spinning mirror light field reconstruction displays in the field of true three-dimensional display. The proposed algorithm describes the correction processing in the procedure of converting the computer three-dimensional model into an image sequence, which required by the light reconstructing 3D display,presents a correction formulation for the emendation of sophisticated distortion of images and gives the image mapping coordinate system at the end of this passage. The experimental result shows that the proposed algorithm enable the correction and reconstruction of three-dimensional display images sequence in high-efficiency and quality.

\section{Introduction}

The spinning mirror light field reconstruction display, one type of volumetric display[1], is based on the principle to display three dimensional objects by reconstructing the light reflected from various direction (shown in Fig.1)[2,3]. We collected two dimensional mapped images from every single angles in the same horizontal position. Then projecting the single frame image to the surface of rotated mirror synchronously[4,5]. However, the distortion appears in the inclination mirror is the biggest problem of the spinning mirror 3D display[6,7]. The correction algorithm is responsible for converting the images sequence of computer models. The innovations of this paper include:

(1) A new Image correction algorithm for generating images source of the spinning mirror display.

(2) The analysis and solution for comprehensive distortion of the image in the procedure of light-field image acquisition and processing.

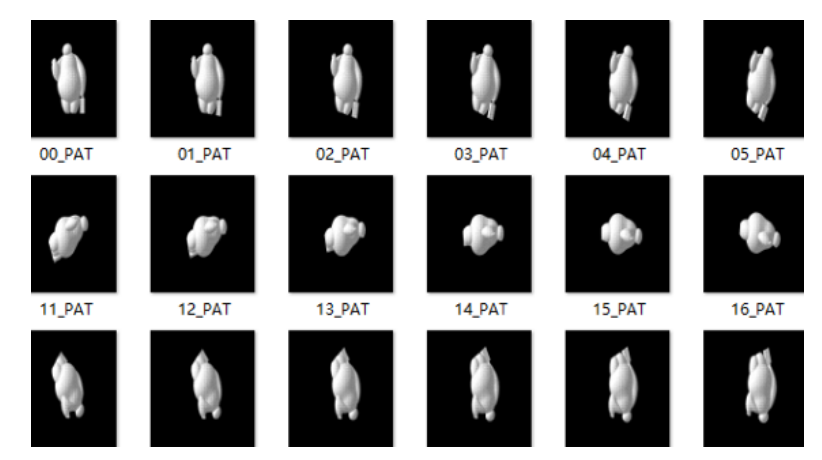

Fig. 1: The images sequence will be sent to projector system and be projected chronologically to comprise 3D image.

Calculating the Factor of Correction. Since the images are projected into an inclination screen by high-speed projector (45 degree will be given for the example), the light of the optical source LED is nonparallel light. In the projective processing, the image will be distorted when appearing in the eyes of people. We coin three concepts to describe the relationship between each processing-the original IO, the distort factor DS and the mirror image IM: 


$$
\mathrm{IO}[\mathrm{U}, \mathrm{V}]=\mathrm{DS}[\mathrm{U}, \mathrm{V}] \times \mathrm{IM}[\mathrm{U}, \mathrm{V}]
$$

Assuming an image $\mathrm{A}$, the outside border of the projecting light named $\mathrm{B}$, the mirror is $\mathrm{C}$. During the processing, $\mathrm{B}$ keep a constantly position and would not change, both $\mathrm{A}$ and $\mathrm{C}$ are changing the direction with the athletic. The irregular quadrilateral distortion will be arisen except the normally trapezoid distortion in this procedure (shown in Fig.2). Luckily, the irregular quadrilateral distortion is the synthesis consequence of trapezoid and single direct elongate distortion caused by the DMD itself, thus the correction factor of the total image could be completed by deriving the factor $\mathrm{K}$ of the trapezoid distortion and the $\mathrm{T}$ of the single direct elongate distortion:

$$
\mathrm{DS}[\mathrm{U}, \mathrm{V}]=\mathrm{K}[\mathrm{U}, \mathrm{V}] \times \mathrm{T}[\mathrm{U}, \mathrm{V}]
$$

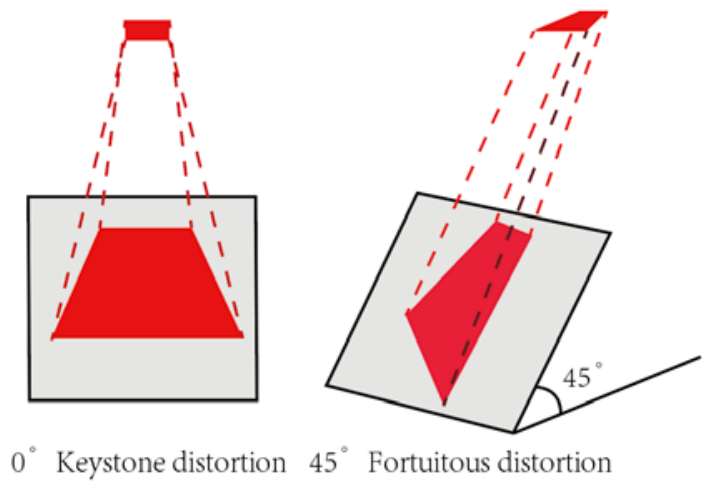

(a)

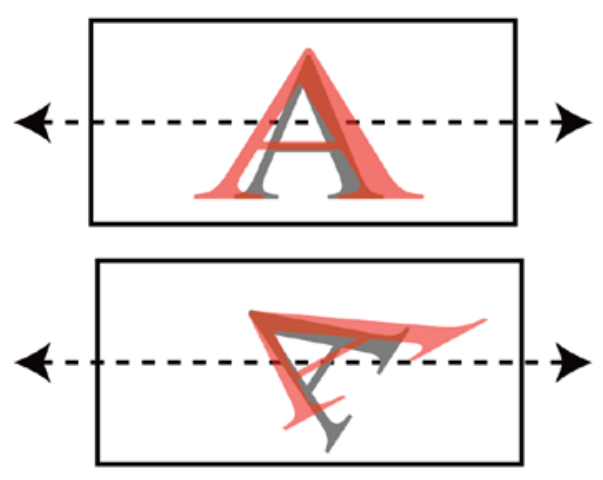

(b)

Fig. 2: (a) The figure illustrates the most common distortions appearing during a rotation period of the mirror, include the keystone distortion and fortuitous distortion. (b) The A shown in grey is the original character and shown in red is the final display effect cause by the single direction stretching of DMD chips.

The correction factor $\alpha$ of keystone distortion and $\beta$ of single direct elongate could be calculated by the factor of $\mathrm{K}$ and $\mathrm{T}$, and distortion of images would be solved. According to the known parameter -original image $\mathrm{IO}(\mathrm{u}, \mathrm{v})$, the observed image $\operatorname{IM}(\mathrm{u}, \mathrm{v})$, the included angle between the ground and the screen, the throw ratio of the projector $\tau$, the distance $\mathrm{H}$ between the exit pupil and the central line in the screen, then the correction factor could be figured out. The distortion factor should be concluded respectively since the transformative factor of $\mathrm{U}$ and $\mathrm{V}$ are different in reason of distorted images differing from each direction.

The single direct elongate distortion caused by the reason of a relativity rotating stretching axis, which elongates the image from every different angle of a circle since the images displayed by the DMD rotating around central axis, but the elongate axis of DMD is constantly kept in one direction. The single direct elongate distortion is raised. For the purpose to finish the correction of this phenomenon, constant constriction is required to be applied on all single frame image in horizontal direction. If the sequence number of an image is $\mathrm{N}$, the resolution of angle is $\mathrm{M}, \mathrm{w}$ and $\mathrm{h}$ are assumed as the weight and the height of the DMD display size. Hence the correction factor $\beta$ of single direct elongate:

$$
\beta=\frac{(M \times N)^{\circ}}{\left(\frac{w}{h}\right)}
$$

The meaning of this formulation is that, in the $\mathrm{M} \times \mathrm{N}^{\circ}$ axis of the picture $\mathrm{N}$, dividing by $\mathrm{w} / \mathrm{h}$ times of this picture. 
Suppose the size of the screen is $\mathrm{L} \times \mathrm{L}$, the inclination angle is $\theta, \mathrm{H}$ is the distance between exit pupil and central line of the screen, the throw ratio $\mathrm{T}$ is 1.66 . Now projecting a square into the screen, the length of DF (central line of square) will not distort since the distance is exactly same with the pure horizontal screen position, the $\mathrm{DF}=\mathrm{H} / \mathrm{T}$. Coincidentally, because the frame shape of projective light is a rectangular pyramid(seen in Fig.3), GI $=D^{\prime} F^{\prime}=(H+L \cdot \sin \theta / 2) / T$, also $A C=(H-L \cdot \sin \theta / 2) / T$, in a similar method. It can be readily seen that the distortion would becoming larger with the mutation of the distance between exit pupil and the screen(seen in fig. 3).

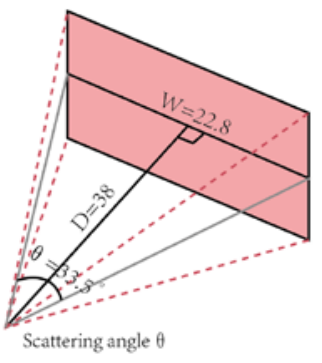

(a)

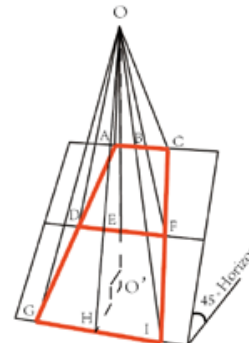

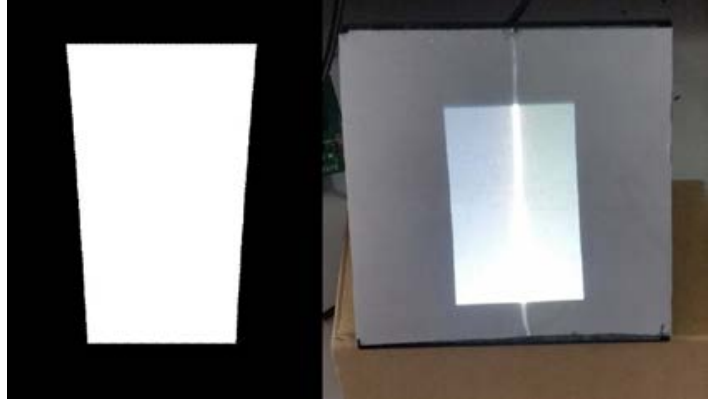

(b)

Fig. 3: (a) The light rectangular pyramid is decided by the Throw ratio( $\mathrm{T}=1.66)$ of the light engine system. Essentially, the trapezoid shown on the screen is the cross section of screen and the light pyramid. According to this theory we could calculate the correction factor. (b) The result of image after correction processing shows the correction factor is properly for the model.

The OE is the central line from the pupil to the middle of the screen, and the length of DF would not be changed. The length of GI and AC changed due to the variation of height with the changed angle $\theta$. This proves the distortion leads to the variation, the greater the difference between height and the central dot, the greater the distortion.

According to the analysis, the linear equation of $A G$ is $y=(-\sin \theta) / T X$, from this equation we could derive the length of image in different position:

$$
\mathrm{L}=\mathrm{H} / \mathrm{T}+\mathrm{X} \sin \theta / \mathrm{T}
$$

So the factor of distortion $\mathrm{K}$ is:

$$
\mathrm{K}=\mathrm{L} /(\mathrm{D} / \mathrm{T}) \text {. }
$$

The correction factor of trapezium distortion $\alpha$ :

$$
\alpha=\mathrm{D} /(\mathrm{T} / \mathrm{L})
$$

Different positions have different correction factors, with the increase of factor $\mathrm{X}$, correction factors changed linearly, and the top of image will be stretched and the bottom of image will be compressed according to equations. Now we have factor $\alpha$ and $\beta$ described in preceding part of passage, the formula factor of correction can be simplified as below:

$$
\operatorname{IR}(\mathrm{U}, \mathrm{V})=\alpha \times \beta \times \mathrm{IO}(\mathrm{U}, \mathrm{V})
$$

Image Mapping Coordinate System. For the purpose to apply the formula in software and convert it to codes of computer practically and conveniently, the image mapping coordinate system should be derived. For instance, the Fig. 4 shows a coordination of point $\mathrm{P}$ in the computer is $\left(\mathrm{x}_{0}, \mathrm{y}_{0}\right)$, in the screen without distortion is $(\mathrm{x}, \mathrm{y})$, in the screen with distortion is $\left(\mathrm{x}_{1}, \mathrm{y}_{1}\right)$, since the computer 
coordination is in different systems with the screen coordination, the converting factor $\alpha$ should be calculated:

$$
\alpha=\frac{\mathrm{P}(\mathrm{x}, \mathrm{y})}{\mathrm{P}\left(\mathrm{x}_{0}, \mathrm{y}_{0}\right)}=\frac{\mathrm{H} / \mathrm{T}}{\mathrm{IW}}
$$

The $\mathrm{H}$ is the distance from the pupil to the screen, $\mathrm{T}$ is the Throw Ratio of the light engine, $\mathrm{H} / \mathrm{T}$ is the length of image in the central screen, IW (img. width) is the weigh in the computer and 608 will be used as the example of the weigh here. Next, the mapped relations of computer standard images versus screen standard images, screen standard images versus screen actually images, the computer standard images versus screen actually images will be derived. The conditions are given below: The DMD stretching length of weight is $w$, the DMD stretching length of height is h, the DMD actual stretching ratio is $\mathrm{w} / \mathrm{h}$.

The first equation is involved to the computer standard images versus screen standard images, if the magnification of images is $\alpha$, the $\mathrm{X}$ direction will be enlarged $\alpha$ times. The $\mathrm{Y}$ direction will form a right angled triangle namely has a $\alpha \times 1 / \cos \theta$ times enlarge.

$$
\left\{\begin{array}{l}
x=\alpha x_{0} \\
y=\frac{\alpha y_{0}}{\cos \theta}
\end{array}\right.
$$

The mapped relation of screen standard images versus screen actually images:

$$
\left\{\begin{array}{c}
\mathrm{x}_{1}=\frac{H-y \sin \theta}{H} \times \frac{W}{h} \times \mathrm{x} \\
y_{1}=y
\end{array}\right.
$$

\section{Correction factor:}

$$
\begin{aligned}
& K_{x}=\frac{H}{\frac{W}{h}(H-y \sin \theta)}=\frac{H}{\frac{W}{h}\left(H-\frac{\alpha y_{0}}{\cos \theta} \sin \theta\right)}=\frac{1}{\frac{W}{h}\left(1-\frac{1}{T \times I W} y_{0} \tan \theta\right)} \\
& \mathrm{K}_{y}=\frac{1}{\tan \theta}
\end{aligned}
$$

For the dot $\mathrm{P}\left(\mathrm{p}_{0}, \mathrm{q}_{0}\right)$ in the standard computer coordination system, the coordination after correction processing is:

$$
\left(\frac{1}{\frac{W}{h}\left(1-\frac{q_{0} \tan \theta}{T \times I W}\right)} p_{0}, \frac{q_{0}}{\tan \theta}\right)
$$

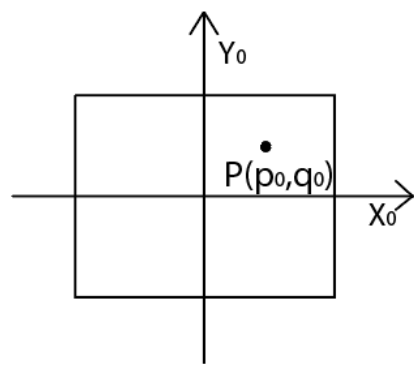

Computer standard images

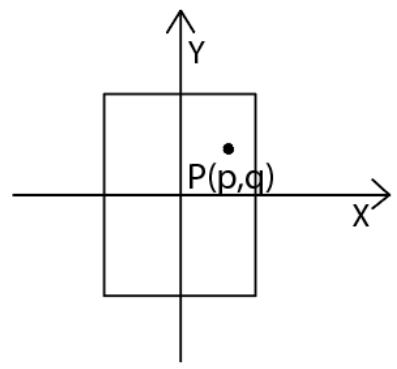

Screen standard images

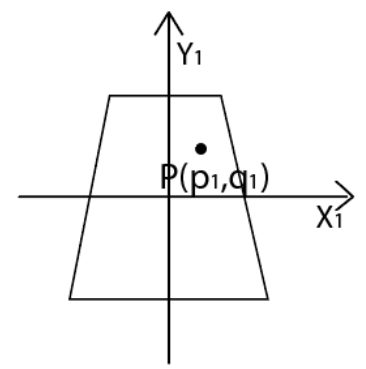

Screen actually distorted images

Fig. 4 The Image mapping coordinate system shows the transformation of a point form computer standard coordination to the final screen coordination . 


\section{Results and Experiments}

Since the upper limit of the refresh rate of the DLP projector is determined by the flipping speed of DMD chips, that is, the image is transformed when the micro mirrors are turned every time, which determines that each pixel of the image can only be represented as a black or white binary image. Therefore, it is necessary to binarize the generated image. The corrected images will be created after all of the processing steps were described above the passage. At last part, we use a prototype display for practical test and applied effect evaluation. The effects are shown in Fig.5.

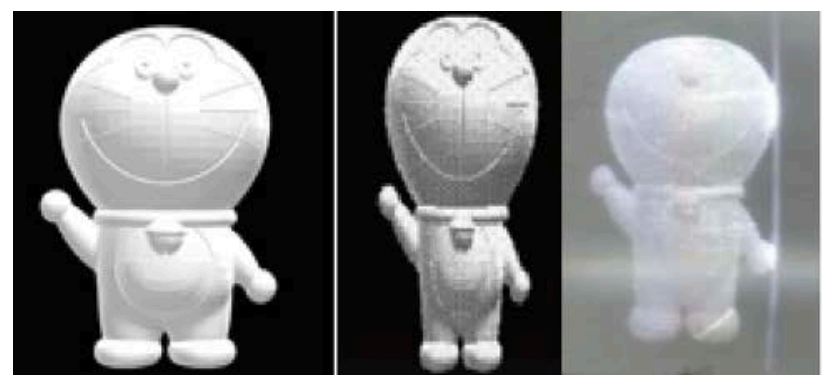

(a)

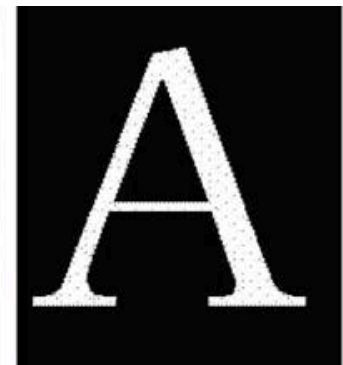

(b)

Fig. 5: (a) The 0-degree original 3D object in the computer, the corrected image and the displayed image (from left to right). (b) The 45-degree original letter in the computer, the corrected image and the displayed image (from left to right). The reconstruction effect is highly similar to the original image proved by the final display experiments.

\section{Conclusions}

The proposed correction algorithm is based on the theory of volumetric spinning mirror display, accomplishes the task of images sequence securing, keystone distortion and fortuitous distortion correction, summarizes the processing of correction by the calculation of mapping image coordination. generates a group of images which are theorically and practically important to improve display quality and expedite calculation speed for the spinning mirror volumetric display.

\section{References}

[1] ARL Travis: Proceedings of the IEEE Vol. 85 (1997), p. 1817

[2] Xinxing Xia: Research on Three - dimensional Display Mechanism and Realization Technology of Horizontal Light Field (Zhejiang University, China 2014). (In Chinese)

[3] Jin-Ho Lee, Juyong Park, Dongkyung Nam, Seo Young Choi, Du-Sik Park, Chang Yeong Kim: Optics Express Vol. 21 (2013), p. 26820

[4] Andrew Jones, Ian McDowall, Hideshi Yamada, Mark Bolas, Paul Debevec: ACM Transactions on Graphics Vol. 26 (2007), p. 40

[5] Qing Zhong: Research on Display Principle and Technology of Multi-projection Light Field Display System (Zhejiang University, China 2016). (In Chinese)

[6] Andrew Jones, Koki Nagano, Jing Liu, Jay Busch, Xueming Yu, Mark Bolas, Paul Debevec: Journal of Electronic Imaging Vol. 23 (2014), p. 011005

[7] Techang Dai, Wenting Zheng, Xinxing Xia: Journal of System Simulation Vol. 10 (2013), p. 2304(In Chinese) 